\title{
Air Pollutant Source Location based on Zigbee Wireless Network and Optimized Least Square
}

\author{
Chengxiang Yin \\ Beijing Institute of Technology, Beijing 100081, China; \\ ycxbit1994@163.com
}

\begin{abstract}
Keywords:air pollutant source location, wireless sensor network (WSN), Zigbee wireless network, optimized least square, cycle pollutant monitoring.
\end{abstract}

\begin{abstract}
This paper proposed an air pollutant source location system with the combination of the wireless sensor network (WSN) technology based on Zigbee wireless network and an optimized least square method. The system, which can realize the coarse location of the possible air pollutant sources, is illustrated in detail from the factors of technology, architecture and working scheme. This paper also proposed an optimized monitoring method applied to each Zigbee node named cycle pollutant monitoring to reduce the amount of transmission data. The paper finally concluded the advantages as well as the disadvantages of the current system, and made a brief statement of two possible optimization directions.
\end{abstract}

\section{Introduction}

With the rapid urbanization and industrialization process in the world, the problems related to the air pollution have become increasingly severe, especially in the modern cities. It has been acknowledged that the air pollution can cause many health-related issues among people from all ages, for the basic reason that the pollutants in the air can be harmful to the respiratory system which is of great importance of people. Also, it has been recognized that air pollutants usually originate from industrial emission and vehicle emission. The original source of the industrial emission can be easily located at the factories; however, the source of the vehicle emission cannot be precise positioning, as it can vary with traffic condition and time. So, air pollutant source location is an urgent issue and needs some new technologies.

This paper proposed an air pollutant source location system based on Zigbee wireless network and an optimized least square method.

\subsection{Overview of Wireless Sensor Network.}

Wireless sensor network (WSN) is defined as a network which has spatially distributed autonomous sensors to monitor physical or environmental conditions, such as temperature, humidity and sound. It can form a multi-hop ad hoc network based on wireless communication. In the location system proposed by this paper, the WSN is realized based on Zigbee technology.

Compared with the similar GPRS sensor network, advantages of WSN are as followed.

Environmental adaptability: For the convenience of the each Zigbee node in WSN, it can be easily deployed in a dense form or a sparse form in a specific area. So in this system, the Zigbee sensor network can cover a large area, and immediately locates the possible source of air pollutant. However, the size of each GPRS node is larger compared with the WSN node, and it will be more difficult to deploy it in some extreme conditions. Also, the GPRS sensor network can only work at the area which is covered by the GPRS signal. But the WSN can be established easily when there exist Zigbee nodes no matter what the condition is.

Stability: For the Ad hoc network characteristic of the WSN, it can organize and manage itself automatically even if one or several Zigbee nodes in WSN have been damaged or paralyzed. This characteristic can guarantee the normal operation of WSN in some extreme conditions. However, as for GPRS sensor network, the communications between the GPRS modules in the same area with the server are all based on the normal operation of the local base station. So, if the base station is knocked off, all the GPRS nodes in the coverage of base station will be paralyzed. 
Low power consumption: The power consumption of each Zigbee node in WSN is lower compared with the GPRS sensor node. Also the Zigbee node can sleep for some time when it does not need to collect or transmit information and it can sign out or be added into the network according to the actual need. This characteristic can maintain the working life of the WSN system. However, as for each GPRS node, the GPRS module should maintain the wireless access to the Internet the whole process and this will cost large power consumption.

The Zigbee nodes in WSN can be divided into three types, end device, router and coordinator.

End device: In charge of the environmental information collection by using sensors and has a relatively low processing power and storage capacity. Also it can receive and deal with the data sent from the other notes.

Router: In charge the Zigbee node's participation into WSN, and has a relatively strong processing capacity and storage capacity. Sometimes it can act as an communication bridge between end device and coordinator.

Coordinator: In charge of the management of WSN and can start up the wireless network. The user of wireless network can control and access the network resource through the coordinator.

The general architecture of WSN is shown as Fig. 1.

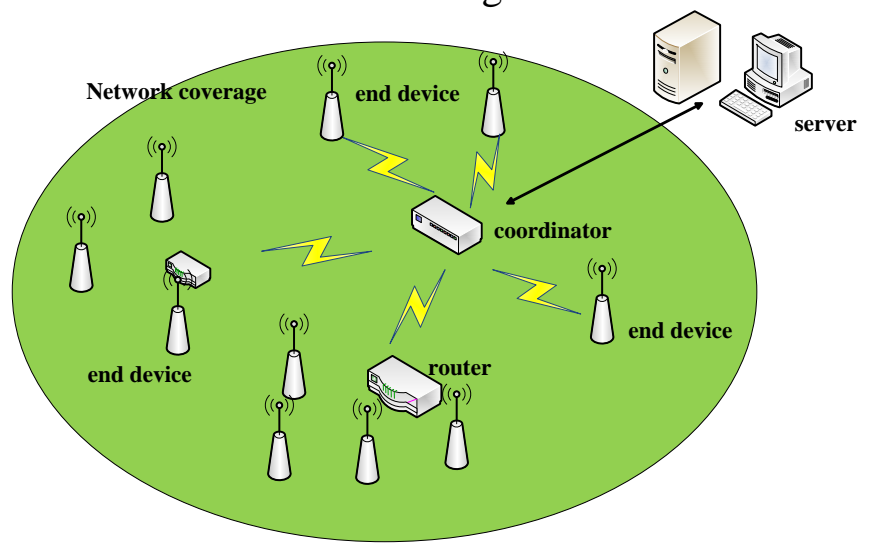

Fig. 1 General architecture of WSN

\subsection{Overview of Pollutant Source Location.}

Technology of WSN be applied into environmental monitoring such as water quality monitoring and forest fire surveillance, and the most obvious contribution of WSN is obtaining large amount of measurement data. However, without knowing the location from where the information is obtained, the measurement is meaningless[1].The pollutant source location also requires the location information of each Zigbee node to be the fundamental statistical data for subsequent analysis.

Much related work about the diffuse source location based on WSN has been done through the last several decades.

Brennan et al. [2] have proposed a distributed sensor network which can detect the radiological dispersion device (RDD) of motor vehicles. Yang et al. [3] have addressed two water pollutant source location methods, based on the least square theory and least absolute deviation, to locate the coastal emission sources, according to the data obtained from WSN. Zhao et al. [4] have proposed an architecture of dynamically querying sensors which is aiming at minimizing the redundancy to increase the information effectiveness.

There exist some similarities between the water pollutant source and air pollutant source, and the similarities are listed as followed.

Uniformly diffused: When the environment remains unchanged and the space is large enough, the pollutants will diffuse in all directions uniformly, no matter in water or in air.

Distribution Law: The pollutants density is inversely proportional to the distance from the pollutant source no matter in water or in air.

Simplification Law: The pollutants have a three-dimensional diffusion no matter in water or in air, and can both be simplified using the two-dimensional diffusion model for practical condition.

So this paper proposed an optimized least square method to locate the air pollutant source, based on the water pollutant source location algorithm and the minimal information transmission theory, 
using the technology of Zigbee wireless network.

\section{System Architecture and Working Scheme}

\subsection{Architecture of Air Pollutant Source Location System.}

The architecture of air pollutant source location system is shown in Fig. 2. The system is divided into two parts - WSN module, Server module.

WSN module: The WSN module in the system is composed of several Zigbee nodes and one coordinator. The function of WSN is to collect the density of each air pollutant in the area covered by WSN. WSN module can be further divided into two parts, the Zigbee nodes and Zigbee coordinator. The function of each Zigbee node in the network is measuring the air pollutant density with controlling of sensors, and obtains the location information, time and data with controlling of a GPS module. The function of Zigbee coordinator in this system is to collect the data from all the Zigbee nodes, which have been connected with the coordinator, and to send the collected data to server using serial communication.

Server module: The server module in our system is composed of serial receiving software, a deployed database and a data analysis program. It can receive the data transmitted by the serial communication from Zigbee coordinator, and store the received information to deploy database according to the node ID and time. The data analysis program will operate continuously to analyze the data received within a certain period to locate the possible pollutant sources.

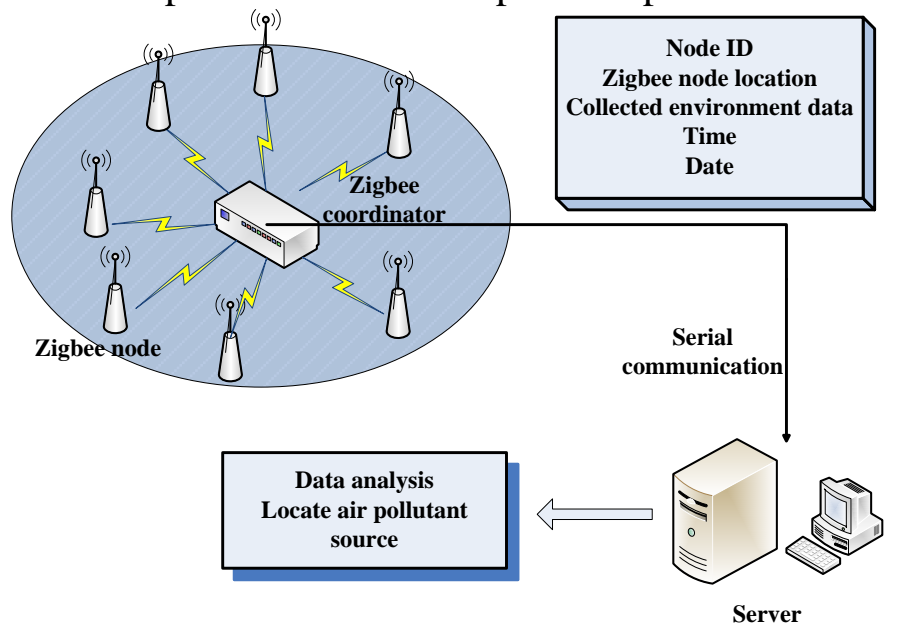

Fig. 2Architecture of air pollutant source locating system

\subsection{Architecture of Zigbee Node.}

The system use CC2530 to act as Zigbee node. The architecture of one Zigbee node is shown in Fig. 3.

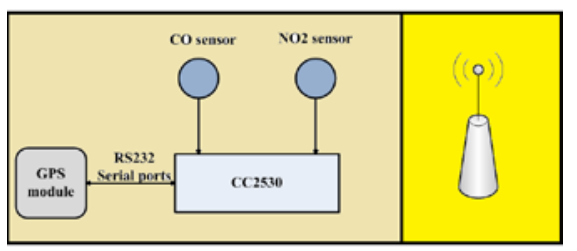

\subsubsection{Overview of CC2530}

Fig. 3Architecture of one Zigbee node

The CC2530 is a single-chip device which has rich built in resources for digital input and output ports, an 8Kbytes RAM and a 64Kbytes flash. It combined the functions of RF transceiver and enhanced 8051 CPU. It is embedded a Zigbee protocol stack named Z-Stack. So it can control the sensors to collect the environmental information based on I/O ports, also can control the GPS module to obtain the information of location and time. Also it can send the collected information to another node using Zigbee wireless network based on Zigbee protocol.

\subsubsection{Overview of GPS module}

The control of GPS module is based on the RS232 serial connection between CC2530 and GPS 
module. The actual connection method is connecting the transmitting port of CC2530 module to the receiving port of GPS module, and connecting the receiving port of CC2530 to the transmitting port of GPS module, and connecting the ground line of CC2530 and GPS module.

The GPS module in the system provides the information of complete location and time. The location information is given in the form of latitude and longitude.

\subsubsection{Information frame}

The system proposed in this paper is aiming at locating the air pollutant source caused by vehicle emission. And it has been proved that the main chemical compositions of vehicle emission are CO and NOx. So, only the CO sensor and the NOx sensor are used in this system to collect the density of the $\mathrm{CO}$ and NOx.

The information collected by one CC2530 node includes latitude, longitude, time, date, CO density and NOx density. In order to guarantee the data integrity, the CC2530 will build an information frame before sending data to coordinator. The information frame is shown in Fig. 4.

\begin{tabular}{|c|c|c|c|}
\hline Node ID & Latitude & Longitude & Time \\
\hline Date & CO density & NOx density & \\
\hline
\end{tabular}

Fig. 4Information frame

\subsubsection{Working flow of Zigbee node}

The working flow of Zigbee node is shown in Fig. 5.

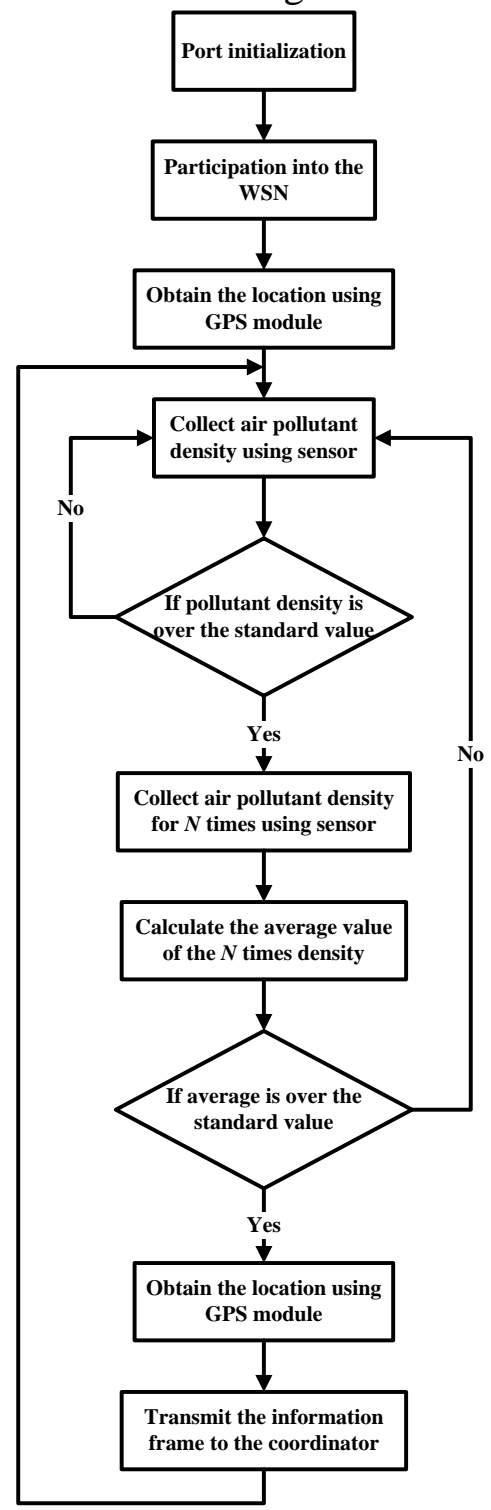

Fig. 5Working flow of Zigbee node

The working flow is realized by embedded programming using $C$ language. The working flow can be divided into four steps, port initialization, participation into WSN, obtain location information and 
cycle pollutant monitoring.

Port initialization: Configure the ports which are related with the sensor data input, and also the serial ports used for controlling of GPS module. After port initialization, the CC2530 can collect air pollutant density from sensors and control the GPS module to obtain information of location and time.

Participation into WSN: This step only completes one work, which is finding the WSN started by a specific Zigbee coordinator, and join in this WSN.

Obtain location information: This step can be seen as one necessary part in the initialization process to obtain the location information given in the form of latitude and longitude. For the Zigbee node in this system will stay at one position once deployed, so the value of latitude and longitude will remain unchanged and can be used in the whole working life of this node.

Cycle pollutant monitoring: This step illustrates the optimization of the information collection and transmission between all Zigbee nodes and the coordinator. First the CC2530 controls the sensors to measure the density of air pollutants every predetermined interval, and compares each measured value with standard value which have been preset. If the density is not larger than the standard value, then continue the normal measurement process; otherwise, measure the density for $\mathrm{N}$ times in a specific time interval and calculate the average value of the $\mathrm{N}$ times density. Then compare the average value with the standard value again, if still larger, then the corresponding information frame must be built up and then sent to the coordinator for the further analysis; otherwise, continue the normal measurement process.

\section{Optimized Least Square Method}

\subsection{Model of Least Square Method for Localization.}

This paper uses the least square method to locate the air pollutant source. To simplify the reasoning process, there are some assumptions which should be made according to the statements in 1.2.

1. The diffusion of air pollutants to all the directions is uniform.

2. The diffusion of the air pollutants can be simplified according to a two-dimensional model.

3. The pollutants density is inversely proportional to the distance from the pollutant source.

4. There exists $M$ Zigbee nodes which are randomly distributed in an area, and the accurate location of each node can be obtained through GPS module. Assume that the positions of $M$ Zigbee nodes is $\left(x_{i}, y_{i}\right)$, and $i=1,2,3 \cdots M$, and the distance between each node and the air pollutant source is $r_{i}$, the air pollutant density at time $t$ for each node is $P_{i, t}$.

5. Assume that the air pollutant source is inside the area covered by WSN which position is $\left(x_{s}, y_{s}\right)$.

6. All the information frames sent by Zigbee nodes can be received by the coordinator, and then be transmitted to the server successfully.

It has been mentioned that the pollutants density is inversely proportional to the distance from the pollutant source, so it can be reasoned that the air pollutant density at each node is:

$$
P_{i, t}=\frac{C}{r_{i}^{\alpha}}+w_{i, t}
$$

where the pollutant density at source is $C$, the power $\alpha$ is related to the environment conditions, the $w_{i, t}$ is the Gaussian noise with zero mean and variance $\sigma_{i, t}$. And $r_{i}$ in this system is:

$$
r_{i}=\sqrt{\left(x_{i}-x_{s}\right)^{2}+\left(y_{i}-y_{s}\right)^{2}}
$$

Based on the reasoning method proposed by Michaelides et al. [5], it can be drawn that the source location $\left(x_{s}, y_{s}\right)$ can be found by minimizing the following function:

$$
\mathrm{Q}=\sum_{\mathrm{i}=1}^{\mathrm{M}}\left(\frac{\mathrm{C}}{\left[\left(\mathrm{x}_{\mathrm{i}}-\mathrm{x}_{\mathrm{s}}\right)^{2}+\left(\mathrm{y}_{\mathrm{i}}-\mathrm{y}_{\mathrm{s}}\right)^{2}\right]^{\frac{\alpha}{2}}}-\overline{\mathrm{P}}_{\mathrm{l}}\right)^{2}
$$

And $\bar{P}_{l}$ is the average value of $N$ times' measurements of air pollutant density and it has a corresponding time in information frame.

\subsection{Optimization of LeastSquare Method.}

The least square method mentioned above will process all the information collected by sensors 
each time, that is to say all the information obtained by the Zigbee nodes will be sent to the server to participate into the least square processing. However, from the perspective of the actual physical meaning, if the air pollutant density collected by a Zigbee node is lower than the standard value, then this density value is very likely to be meaningless because it is less correlated with the air pollutant source. Only the air pollution density which is larger than the standard value is likely to be meaningful.

In this paper, the system first finds the possible meaningful air pollutant density using the cycle pollutant monitoring method mentioned in 2.2.4, and then sends the corresponding information frame to server through the Zigbee wireless network and serial communication. Only the information received by server will participate in the least square processing to locate the air pollutant source. The operation data for each least square processing is the information frame received by the server from Zigbee sensor network in a specific time span. This method can not only largely save the channel bandwidth and minimize the energy consumption caused by the data transmission, but can also reduce the calculation amount in the sever caused by the least square processing. Finally, the least square method is optimized from the perspective of the computation amount.

\section{Conclusion}

\subsection{SystemAdvantages.}

From the perspective of WSN, there exist four advantages, bandwidth saving, energy reduction, large area coverage, and working stability.

Bandwidth saving: Because the paper proposed a cycle pollutant monitoring method in each Zigbee mode, which can largely reduce the amount of transmission data, so the channel bandwidth can be saved.

Energy reduction: For the amount of the transmission data in unit time is reduced, the energy consumption caused by data transmission in each Zigbee node per unit time will be large reduced, then the working life of WSN can be extended.

Large area coverage: Because the size of each Zigbee node is small compared with other microprocessor and its low cost, so the WSN built by Zigbee nodes can be easily deployed and cover a large area.

Working stability: An alternate Zigbee node can be added into the WSN whenever there is a Zigbee node which has been participated into the network is damaged or paralyzed. So the WSN can maintain the stability by preset some alternate Zigbee nodes inside the coverage area.

From the perspective of the server, there exist two advantages, computation reduction and storage saving.

Computation reduction: Because the paper proposed a cycle pollutant monitoring method which can largely reduce the amount of receiving data at the server, so the amount of data participated into the least square processing will be minimized largely. This will save the computing power of the server and extent the working life of the server.

Storage saving: The amount of data received and then stored into the database deployed in server is reduced, so the server storage can be saved for the use of more effective information.

Based on the above six advantages from the perspective of WSN and server, the system can be easily extended to apply into a larger monitoring area in practical.

\subsection{Optimization Directions of System.}

There exist two main limitations in the current system. The first limitation is that the system cannot realize the precise positioning of the air pollutant source, for all the Zigbee nodes in WSN are remained at a static state and have to cooperate to remotely estimate the air pollutant source. The second limitation is that the system only considers the Gaussian noise with zero mean in the least square processing, and it may not be accurate for the practical conditions.

The two corresponding optimization directions of the system are as follows.

- The system proposed in this paper is suitable for the conditions which have low requirements of the air pollutant source location accuracy, and it can determine a possible area where the air pollutant source might locate in. So the optimized direction of the system is first identifying a 
small area where the air pollutant source may be located in, and then combine with the moving Zigbee nodes to pinpoint the exact location of air pollutant source.

- Replace the Gaussian noise with zero mean with the chi-square noise model or lognormal noise model which is more close to the real environmental conditions of air.

\section{References}

[1]. Mao G, FidanB, Anderson B D O. Wireless sensor network localization techniques[J]. Computer networks, 2007, 51(10): 2529-2553.

[2]. Brennan S M, Mielke A M, Torney D C, et al. Radiation detection with distributed sensor networks[J]. Computer, 2004, 37(8): 57-59.

[3]. Jun Y, Li C, Xu L. Pollution source localization in lake environment based on wireless sensor networks[C]. Control Conference (CCC), 2012 31st Chinese. IEEE, 2012: 6649-6654.

[4]. Zhao F, Shin J, Reich J. BInformation-driven dynamic sensor collaboration for tracking applications,IEEE Signal Process[J]. 2002.

[5]. Michaelides M P, Panayiotou C G. Plume source position estimation using sensor networks[C]. Intelligent Control, 2005. Proceedings of the 2005 IEEE International Symposium on, Mediterrean Conference on Control and Automation. IEEE, 2005: 731-736.

[6]. Al-Ali A R, Zualkernan I, Aloul F. A mobile GPRS-sensors array for air pollution monitoring[J]. Sensors Journal, IEEE, 2010, 10(10): 1666-1671.

[7]. Zhi-gang H, Cai-hui C. The application of zigbee based wireless sensor network and GIS in the air pollution monitoring[C]. Environmental Science and Information Application Technology, 2009. ESIAT 2009. International Conference on. IEEE, 2009, 2: 546-549.

[8]. Xu L, Ren X, You Z, et al. Wireless Sensor Network Based Chicken Coop Surveillance System[J]. International Journal of Online Engineering (iJOE), 2014, 10(3): pp. 37-41.

[9]. Yick J, Mukherjee B, Ghosal D. Wireless sensor network survey[J]. Computer networks, 2008, 52(12): 2292-2330.

[10]. Wang W, Yuan Y, Ling Z. The research and implement of air quality monitoring system based on zigbee[C]. Wireless Communications, Networking and Mobile Computing (WiCOM), 2011 7th International Conference on. IEEE, 2011: 1-4.

[11]. Lanjewar U M, Shah J J. Air pollution monitoring \& tracking system using mobile sensors and analysis of data using data mining[J]. International Journal of Advanced Computer Research, 2012, 2(4): 19-23.

[12]. Lu Z, Li Y, Fu T. Intelligent Wind-speed Monitoring System Based on the Zigbee Wireless Network[J]. International Journal of Online Engineering (iJOE), 2015, 11(1): pp. 57-60. 